\title{
Efficacy and Safety of Electroacupuncture for Insomnia Disorder: A Multicenter, Randomized, Assessor-Blinded, Controlled Trial
}

This article was published in the following Dove Press journal:

Nature and Science of Sleep

\author{
Boram Lee' \\ Bo-Kyung Kim ${ }^{2}$ \\ Hyeong-Jun $\mathrm{Kim}^{3}$ \\ In Chul Jung $\mathbb{D}^{4}$ \\ Ae-Ran Kim (iD) \\ Hyo-Ju Park' \\ O-Jin Kwon (D) \\ Jun-Hwan Lee $\mathbb{D}^{1,5}$ \\ Joo-Hee $\mathrm{Kim}^{6,7}$
}

'Clinical Medicine Division, Korea Institute of Oriental Medicine, Daejeon, Republic of Korea; ${ }^{2}$ Department of Neuropsychiatry, School of Korean Medicine, Pusan National University, Yangsan-si, Gyeongsangnam-do, Republic of Korea; ${ }^{3}$ Department of Oriental Gynecology, Jecheon Oriental Hospital of Semyung University, Jecheon, Republic of Korea; ${ }^{4}$ Department of Neuropsychiatry, College of Korean Medicine, Daejeon University, Daejeon, Republic of Korea; ${ }^{5}$ Korean Medicine Life Science, University of Science \& Technology (UST), Campus of Korea Institute of Oriental Medicine, Daejeon, Republic of Korea;

${ }^{6}$ Department of Acupuncture and Moxibustion Medicine, College of Korean Medicine, Sangji University, Wonju-si, Gangwon-do, Republic of Korea;

${ }^{7}$ Research Institute of Korean Medicine, Sangji University, Wonju-si, Gangwon-do 26339, Republic of Korea

Correspondence: Joo-Hee Kim Department of Acupuncture and Moxibustion Medicine, College of Korean Medicine, Sangji University, 83, Sangjidaegil, Wonju-si, Gangwon-do, Republic of Korea

Tel +82 337419268

Fax +82 33 74l 914I

Email jhkim7|4v@gmail.com
Purpose: To evaluate the efficacy and safety of electroacupuncture in treating insomnia.

Patients and Methods: In a multicenter, randomized, assessor-blinded, controlled trial, 150 patients with DSM-5-diagnosed insomnia with Insomnia Severity Index (ISI) scores $\geq 15$ were randomly assigned to three different groups that underwent 10 sessions of electroacupuncture, sham-electroacupuncture, or usual care for 4 weeks from October 2015 to June 2016 at four Korean medicine hospitals, Republic of Korea. The primary outcome included the ISI score at Week 4; the secondary outcomes included evaluations of Pittsburgh Sleep Quality Index (PSQI), sleep diary, Hospital Anxiety and Depression Scale (HADS), EuroQoL five dimension (EQ-5D), Patient Global Impression of Change (PGIC), and salivary melatonin and cortisol levels. Assessments were performed at baseline (Week 0) and at Weeks 2, 4, 8, and 12.

Results: Compared with the usual care group, electroacupuncture group showed a greater improvement in ISI, PSQI, sleep diary-derived variables and HADS and EQ-5D scores at Week 4 . The effects mostly persisted until Week 12 . There were no significant differences between electroacupuncture and sham-electroacupuncture groups at Week 4 in all outcome measures, except sleep diary-derived sleep efficiency. However, the ISI score showed a significant difference between these groups at Weeks 8 and 12. Treatment success as per PGIC was significantly and borderline higher for electroacupuncture compared with usual care and sham-electroacupuncture, respectively. No significant changes in salivary melatonin and cortisol levels before and after treatment were observed in all groups. No serious adverse events were reported. Blinding was maintained in the sham-electroacupuncture group.

Conclusion: Ten sessions of electroacupuncture can improve the sleep quality of patients with insomnia without serious adverse effects. Thus, it can be recommended as an effective, safe, and well-tolerated intervention.

Keywords: electroacupuncture, insomnia, sleep initiation and maintenance disorders, randomized controlled trial

\section{Introduction}

Insomnia is one of the most frequent sleep disorders, characterized by difficulty in initiating or maintaining sleep and/or an inability to return to sleep after waking up too early, lasting for at least 3 months. ${ }^{1}$ The prevalence of insomnia in the general population varies depending on the geographical location, and ranges from $6 \%$ to $50.5 \%$. $^{2,3}$ Insomnia not only decreases the quality of life but also causes adverse outcomes such as increased risks of diabetes, cardiovascular disorders including hypertension and myocardial infarction, and even suicidal thoughts and behaviors. ${ }^{4-7}$ 
Therefore, early diagnosis and effective treatment of insomnia are very important for public health.

Generally, cognitive behavioral therapy for insomnia (CBT-I) supplemented with short-term hypnotics is recommended as first-line treatment for insomnia patients. ${ }^{8,9}$ However, CBT-I cannot meet the population needs given the lack of a sufficient number of therapists and limitations of time or insurance. Further, $25 \%-40 \%$ of insomnia patients do not achieve remission by CBT-I. ${ }^{10,11}$ In addition, hypnotics are associated with diverse adverse effects such as residual daytime sedation, dizziness, drowsiness, nausea, mood changes, cognitive impairment, motor incoordination, and dependence. ${ }^{12-14}$ Because of these limitations of conventional medicine, the use of complementary alternative medicine (CAM) for treating insomnia has increased from $20.6 \%$ in 1990 to $45 \%$ in 2007 in the United States. ${ }^{15,16}$

Acupuncture, one of the most popular CAM modalities, has been used for treating insomnia since antiquity in East Asia and is now widely accepted in Western countries. ${ }^{17}$ Electroacupuncture, a modified form of conventional acupuncture, stimulates acupoints by electrical stimulation via inserted acupuncture needles. ${ }^{18}$ Because the body tissue is a type of an electrical conductor, it is believed that electrical impulses can strengthen stimulation through the needles at acupoints. A recent network meta-analysis comparing the effectiveness of different forms of acupuncture in the treatment of insomnia showed that electroacupuncture alleviated insomnia more significantly compared with the conventional acupuncture. ${ }^{19}$ Many clinical trials have been conducted on acupuncture and electroacupuncture for insomnia; however, definite conclusions cannot be drawn about the effects of these measures because of small sample sizes and the poor methodological quality of published studies. ${ }^{20-22}$ Moreover, recent clinical practice guidelines for the management of chronic insomnia have referred to acupuncture, stating that there is insufficient evidence to conclude the efficacy and safety of acupuncture for treating insomnia. ${ }^{23}$

Therefore, conducting large-scale, high-quality randomized controlled trials (RCTs) evaluating the efficacy of electroacupuncture in treating insomnia, with appropriate and validated placebo control, is important for confirming its effects. Thus, the main objective of our study was to evaluate the efficacy and safety of electroacupuncture in patients with insomnia and compare the outcomes with those observed for sham-electroacupuncture or usual care.

\section{Patients and Methods}

\section{Study Design}

This multicenter, randomized, assessor-blinded, controlled trial was conducted in accordance with the Declaration of Helsinki from October 2015 to June 2016 at the following four Korean medicine hospitals in the Republic of Korea: Daejeon Korean Medicine Hospital of Daejeon University, Dunsan Korean Medicine Hospital of Daejeon University, Dongeui University Korean Medical Hospital, and Semyung University Korean Medicine Hospital. The investigators randomly assigned participants for 10 sessions (2-3 times a week, for 4 weeks) in either one of the following three groups: electroacupuncture, shamelectroacupuncture, or usual care group. The study was approved by the institutional review board at each site (approval numbers: DJOMC-128, DJDSKH-15-06-1, 2015-03, and 2015-08-02). Written informed consent was obtained from all participants before study commencement. The protocol of this trial was registered with the Clinical Research Information Service (registration number: KCT0001685) and a detailed version of the study methodology has been published. ${ }^{24} \mathrm{We}$ followed the Consolidated Standards of Reporting Trials (CONSORT) and Standards for Reporting Interventions in Clinical Trials of Acupuncture (STRICTA) recommendations while designing and reporting this trial. ${ }^{25,26}$

\section{Participants}

Participants were enrolled through advertisement in the local newspaper and hospital websites and those who met the eligibility criteria and voluntarily signed the informed consent form were recruited. Participants aged 19-64 years, who were diagnosed with insomnia (ie, having difficulty in initiating or maintaining sleep, or early awakening, at least three times a week for 3 months or longer) as per Diagnostic and Statistical Manual of Mental Disorders, Fifth Edition (DSM-5) by a Korean medicine doctor and had an Insomnia Severity Index (ISI) score of at least 15 , were included in the study. However, if they fulfilled even one of the following criteria, they were excluded: other psychiatric disorders such as major depressive disorder, anxiety disorder, and/or panic disorder; caffeine, alcohol, or drug addiction; depression score of Hospital Anxiety and Depression Scale (HADS) score $\geq$ 11 points; unregulated hormone disorder; serious or systemic disease such as hyperthyroidism that could affect sleep; pain or any disease that could cause insomnia; 
previous therapy or medication for insomnia in the past 2 weeks or change in the type or dosage of a regularly administered medication to alleviate insomnia in the past 4 weeks; working shifts or changes in day/night work schedule that could affect the circadian rhythm; administration of blood coagulants for cardiovascular or hemostatic disorders; hypersensitivity with regard to acupuncture or difficulty in co-operation with acupuncture therapy; presence of implants that could interfere with electroacupuncture or a history of hypersensitivity to electrostimulation; pregnancy, lactation, or pregnancy planning; and difficulty in compliance with the study protocol because of issues such as infection, abscess, skin lesions, or other skin diseases close to the site of selected acupoints.

\section{Randomization and Blinding}

An independent statistician generated a random sequence number using SAS version 9.4 software (SAS Institute Inc., Cary, NC, USA), with an allocation ratio of $1: 1: 1$ to the three groups, stratified by whether conventional medications for insomnia are being administered and the institutions conducting the trial using block randomization. Sealed opaque envelopes were used for allocation concealment. The envelopes were opened by the practitioners at the baseline visit of the enrolled participants, and the group allocation was blinded to other related researchers during the study period. Blinding was maintained between the real electroacupuncture and sham-electroacupuncture groups throughout the study period, and a blinding test was conducted to evaluate whether blindness was maintained. As it was impossible to blind the practitioners and participants allocated to the usual care group given the nature of the intervention, outcome assessors and data analysts who were not involved in the treatment procedures were blinded to this group allocation.

\section{Intervention}

The intervention was initiated after randomization within 2 weeks of the screening visit in participants who met all inclusion criteria and did not meet any exclusion criteria. In the electroacupuncture group, disposable sterilized stainless steel filiform needles $(0.25 \times 40 \mathrm{~mm}$; Dongbang Acupuncture Inc., Seoul, Republic of Korea) were inserted at standardized GV20, EX-HN3, and bilateral HT7, PC6, BL63, and KI4 acupoints with a 5-20 mm depth of insertion using 10 acupuncture needles (one needle for each acupoint). Acupoint selection was based on previous electroacupuncture studies conducted for insomnia, textbooks on acupuncture and moxibustion, and consensus between Korean medicine specialists. $^{20,21,27-29}$ The manipulation technique was applied at the acupoints until the "de qi" sensation (soreness, numbness, heaviness, and distention) was achieved in participants. The treatment was performed 2-3 times a week for 4 weeks, with a total of 10 sessions. In the sham-electroacupuncture group, a Park Sham Device (PSD; Dong-bang AcuPrime Ltd., Exeter, UK), a frequently used sham acupuncture device with a blunt end that does not penetrate the skin, ${ }^{30,31}$ was inserted at 10 specific non-acupoints on both the arms and legs. These non-acupoints have no therapeutic effect according to Korean medicine theory: "Arm 1" (mid maximal ridge of biceps brachii muscle belly), "Arm 2" (1.5 cm upward to Arm 1), "Leg 1" (1.5 cm upward to the middle of the patella), "Leg 2" (upper $1 / 3$ of the medial part of tibia), and "Leg 3 " $(1.5 \mathrm{~cm}$ lower to Leg 2). Other treatment procedures were the same as the electroacupuncture group. In both the electroacupuncture and sham-electroacupuncture groups, an electroacupuncture device (ES-160, Ito Co. Ltd., Tokyo, Japan) was used for electrostimulation for $30 \mathrm{~min}$. Continuous-wave electrostimulation at a frequency of $4 \mathrm{~Hz}$ and $80 \%$ intensity was applied in the electroacupuncture group. However, in the shamelectroacupuncture group, a de-activated electroacupuncture device was connected to PSD with no electrostimulation, but it made the same beeping sound and light indications when operated. Any additional treatment for insomnia was inhibited during the study period in the electroacupuncture and shamelectroacupuncture groups and all treatment procedures were performed by licensed Korean medicine doctors with more than 2 years of clinical experience and more than 6 years of regular medical education. The acupuncture practitioners limited unnecessary conversations, which were not related to the treatment, with the participants. In the usual care group, any treatment for insomnia except Korean medicine treatment such as acupuncture and herbal medicine was allowed and all concomitant treatments were recorded in a case report form (CRF). All study participants in all three groups received sleep hygiene education using a brochure (Table S1).

\section{Outcomes}

The primary outcome was the ISI score, a validated, selfrating 7-item questionnaire assessing the severity of insomnia and associated daily life disturbances. ${ }^{32}$ Total ISI scores ranged from 0 to 28 , and were interpreted as follows: no clinically significant insomnia (0-7), subthreshold insomnia (8-14), moderate insomnia (15-21), and severe insomnia $(22-28) .^{32}$ To target patients with 
moderate-to-severe insomnia, we set a cut-off for ISI score as 15 or higher as the inclusion criterion. A validated Korean version of ISI score was measured at the screening visit, and at Weeks 2 (mid-point), 4 (treatment termination), 8 (follow-up after the final intervention), and 12 (follow-up after the final intervention). ${ }^{33}$

Secondary outcome measures included the following: (1) the Pittsburgh Sleep Quality Index (PSQI) score, (2) sleep diary-derived variables, (3) HADS score, (4) EuroQoL five dimension (EQ-5D) score, (5) Patient Global Impression of Change (PGIC) score, and (6) salivary melatonin and cortisol levels. PSQI is a self-administered questionnaire used to evaluate sleep disturbances within the past month and measure subjective sleep quality, sleep latency, sleep duration, habitual sleep efficiency, sleep disturbances, sleeping medication use, and daytime dysfunction. $^{34,35}$ A final score of $>5$ out of 21 indicates significant sleep disturbance. ${ }^{34}$ ISI directly assesses the perceived severity of insomnia, and the associated functional impairment, whereas PSQI assesses general sleep disturbances. $^{21}$ Because we targeted patients with DSM5-diagnosed insomnia, we chose ISI score rather than PSQI score (at Week 4) as the primary outcome measure.

The sleep diary was used to maintain data concerning the bedtime at night and the waking time in the morning, from which the total time in bed (TIB) was calculated. It also contained data on sleep onset latency (SOL), waking after sleep onset (WASO) during the night, and total sleep time (TST). We calculated sleep diary-derived sleep efficiency (SE) using the formula TST/TIB $\times 100 \%{ }^{9}$ We compared the mean changes in sleep parameters included in the sleep diary during 1 week before the visit.

HADS is a 14-item questionnaire widely used to assess changes in the emotional state of anxiety and depression in patients with chronic diseases, with high scores indicating severe symptoms. ${ }^{36}$ The EQ-5D measures health-related quality of life, and consists of five questions about mobility, personal care, daily activities, pain/discomfort, and anxiety/ depression. ${ }^{37,38}$ It also assesses global health using EuroQoL visual analogue scale (EQ-VAS), with scores ranging between 100 (best imaginable health) and 0 (worst imaginable health). We used PGIC to evaluate the participant's selfperception of overall improvement after treatment using a 7-point Likert scale, ranging from "very much worse" to "very much improved". 39 For PGIC, treatment success was defined as the percentage of "much improved" or "very much improved". Melatonin levels usually increase at night, whereas cortisol levels increase in the morning in normal condition. ${ }^{27,40}$ Therefore, we assessed the levels of salivary melatonin and cortisol at baseline and Week 4 to determine changes in the physiological levels of these variables after treatment.

Further, we assessed adverse events (AEs) during the study period based on medical examinations and laboratory tests including liver, renal, and thyroid function tests and complete blood cell count. The types of AEs commonly observed after electroacupuncture include nervous system disorders such as vertigo and unconsciousness, general disorders and administration site conditions such as pallor, skin and subcutaneous tissue disorders such as skin pigmentation, gastrointestinal disorders such as nausea and vomiting, and cardiac/vascular disorders such as cyanosis and palpitations. ${ }^{41}$ If such AEs were judged to have a causal relationship with the electroacupuncture treatment based on the time interval between the treatment and the appearance of the AE, it was judged as AE related to the electroacupuncture. The severity and causal relationships between AEs and the intervention were also recorded in the CRF. In addition, a blinding test was performed in the electroacupuncture and sham-electroacupuncture groups after the first and last treatments to check whether the blinding of participants in these groups was maintained. For this, we asked the participants about the type of acupuncture they had received and their thoughts were recorded as follows: "real electroacupuncture," "shamelectroacupuncture," or "unknown". We then evaluated the blinding success using the new blinding index (BI) and $95 \%$ confidence interval $(\mathrm{CI}){ }^{42}$

\section{Sample Size and Statistical Analysis}

This study aimed to compare the mean changes in the ISI score at Week 4 between electroacupuncture and usual care groups and between electroacupuncture and shamelectroacupuncture groups. The differences in ISI scores were expected to be larger between the electroacupuncture and usual care groups compared with those between the electroacupuncture and sham-electroacupuncture groups. Based on previous studies on electroacupuncture for insomnia using ISI as the primary outcome measure, ${ }^{20,21}$ a mean difference of 4.15 between electroacupuncture and shamelectroacupuncture and a standard deviation of 6.0 was used for sample size calculation, with a statistical power of $80 \%$ and a two-sided significance level of $2.5 \%$. Therefore, assuming that a study comparing electroacupuncture and usual care requires fewer samples than that comparing electroacupuncture and sham-acupuncture, we chose a larger 
sample size of 40 participants per group to test both hypotheses. Finally, after considering a $20 \%$ drop-out rate, a total of 150 participants (50 participants per group) were recruited.

All statistical analyses were conducted by a statistician who was independent of intervention and evaluation using SAS version 9.4 software (SAS Institute Inc., Cary, NC, USA). Intention-to-treat analysis was conducted for participants who had received at least one treatment and assessment after randomization, and if necessary, a per-protocol analysis was conducted. A two-sided test with a significance level of 0.025 was performed using an analysis of covariance (ANCOVA) with baseline as the covariate and the treatment group as the fixed factors. Dunnett's test was used for inter-group comparisons to compensate for multiple comparisons and a repeated measure analysis of variance was used to assess the differences between groups over time. A paired $t$-test or Wilcoxon signed rank test was used to compare results before and after treatment within groups. Multiple imputations were applied for missing values.

\section{Results}

\section{Participants' Characteristics}

A total of 193 participants were screened for eligibility; of these, 150 were randomly assigned to one of the three groups. Of the included participants, 136 completed the study, whereas the remaining 14 dropped out. Eight participants withdrew their consent to participate in the trial, five violated the study protocol, and one violated the study protocol and withdrew consent (Figure 1). None of the participants withdrew because of AEs. There was no statistically significant difference in demographic and clinical characteristics among the three groups $(\mathrm{P}>0.05)$, except gender ratio $(\mathrm{P}=0.0253)$ (Table 1). In the usual care group, the proportion of females was significantly lower and that of males was significantly higher than those in the other two groups. Further, in the usual care group, only two patients started a new treatment for insomnia during the study period, and each of them was administering dietary supplements and Zolpidem.

\section{Primary Outcomes}

We compared the mean ISI scores between the three groups at Weeks 2, 4, 8, and 12 by ANCOVA. There were significant differences between electroacupuncture and usual care groups throughout the study period $(\mathrm{P}<0.0001$, all). There was no difference in the mean ISI scores at Weeks 2 and 4 between electroacupuncture and sham-electroacupuncture groups $(\mathrm{P}=0.6404,0.0866)$. However, on conducting follow-up assessments at Weeks 8 and 12, the ISI scores were significantly different between the two groups (Week 8: $\mathrm{P}=0.0213$, Week 12:

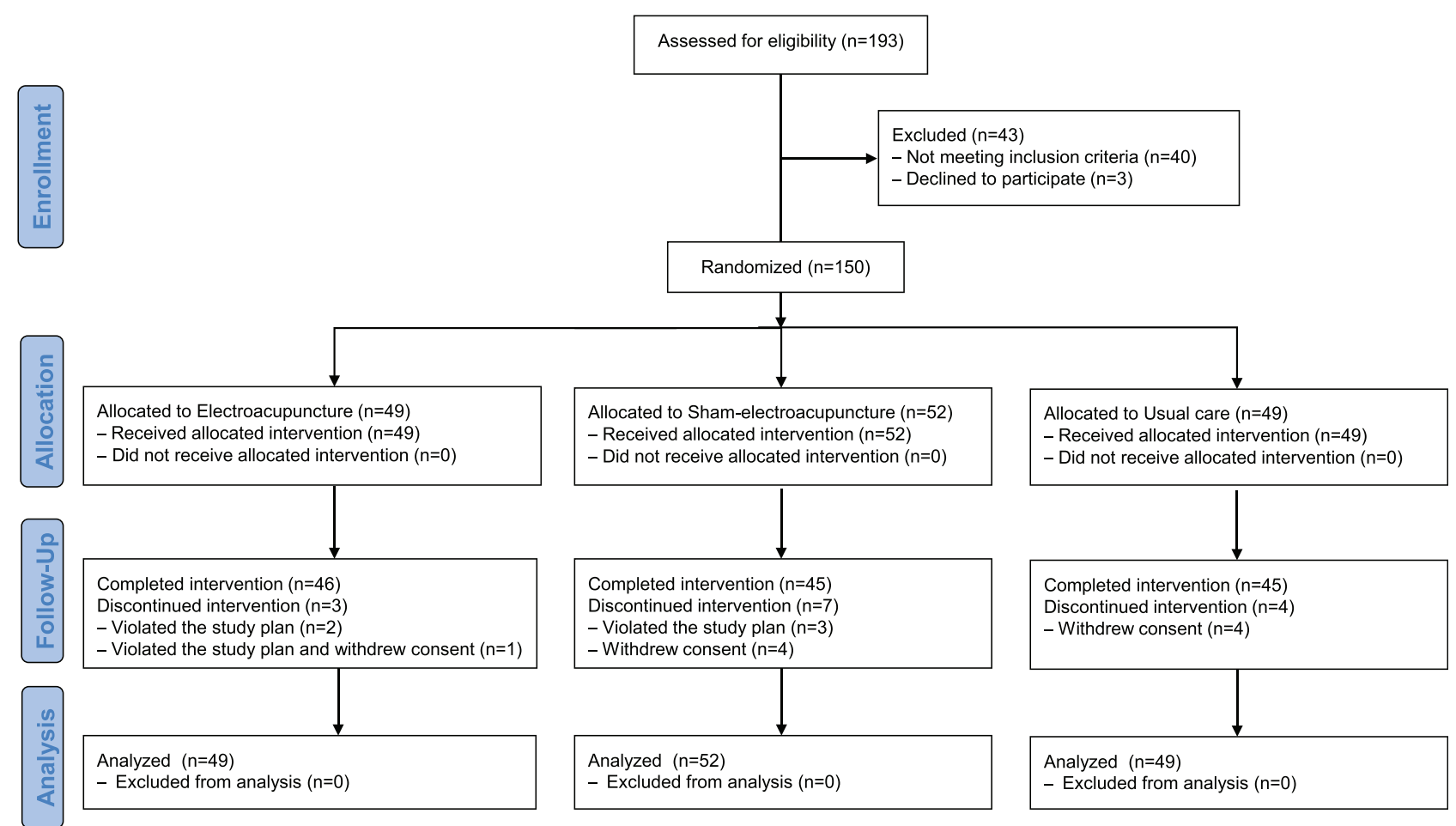

Figure I Study flowchart. 
Table I Demographic and Clinical Characteristics of the Participants

\begin{tabular}{|c|c|c|c|c|}
\hline Characteristics $^{\mathbf{a}}$ & Electroacupuncture $(n=49)$ & Sham $(n=52)$ & Usual Care $(n=49)$ & $P$ value \\
\hline Gender (Male/Female) ${ }^{b}$ & 9 (I8.37\%)/40 (81.63\%) & 9 (17.31\%)/43 (82.69\%) & $19(38.78 \%) / 30(61.22 \%)$ & 0.0253 \\
\hline Age $(\text { year })^{c}$ & $51.78(49.23,54.32)$ & $52.00(49.52,54.48)$ & $52.00(49.48,54.52)$ & 0.9894 \\
\hline Height $(\mathrm{cm})^{\mathrm{c}}$ & $160.5 \mid(158.09,162.93)$ & $159.59(|57.67,16| .5 \mid)$ & $163.56(160.48,166.63)$ & 0.0647 \\
\hline Weight $(\mathrm{kg})^{\mathrm{c}}$ & $59.88(56.98,62.78)$ & $59.65(56.41,62.90)$ & $62.50(58.95,66.04)$ & 0.3884 \\
\hline BMI $\left(\mathrm{kg} / \mathrm{m}^{2}\right)^{\mathrm{c}}$ & $23.12(22.42,23.81)$ & $23.35(22.28,24.42)$ & $23.30(22.18,24.42)$ & 0.9406 \\
\hline Smoke $\left(\right.$ Yes/No) ${ }^{b}$ & $2(4.08 \%) / 47(95.92 \%)$ & $3(5.77 \%) / 49(94.23 \%)$ & $3(6.12 \%) / 46(93.88 \%)$ & 0.9999 \\
\hline Drink $(\mathrm{Yes} / \mathrm{No})^{\mathrm{b}}$ & $19(38.78 \%) / 30(61.22 \%)$ & $15(28.85 \%) / 37$ (7I.15\%) & $22(44.90 \%) / 27(55.10 \%)$ & 0.2304 \\
\hline Exercise $(\mathrm{Yes} / \mathrm{No})^{\mathrm{b}}$ & $12(24.49 \%) / 37(75.51 \%)$ & $16(30.77 \%) / 36(69.23 \%)$ & II (22.45\%)/38 (77.55\%) & 0.6372 \\
\hline Treatment expectation ${ }^{c, d}$ & $6.86(6.43,7.28)$ & $6.56(6.12,6.99)$ & $6.29(5.91,6.66)$ & 0.1561 \\
\hline Duration of symptom (Month) ${ }^{c}$ & $76.37(53.54,99.19)$ & $72.35(53.59,91.10)$ & $82.22(55.85,108.60)$ & 0.8237 \\
\hline
\end{tabular}

Notes: ${ }^{a}$ Data are presented as mean and $95 \%$ confidence interval or number (\%). ${ }^{b}$ Fisher's exact test. ${ }^{\mathrm{c} A n a l y s i s}$ of variance test. ${ }^{\mathrm{d} S e l f-r e p o r t}$ questionnaires are used on a scale of $\mathrm{I}$ to 9 , with higher scores indicating higher expectations.

Abbreviation: BMI, body mass index.

$\mathrm{P}=0.0446$ ). The mean ISI score significantly decreased compared with baseline in all three groups during the treatment period and the scores continued to decrease during the 8-week follow-up period after the final intervention (Figure 2, Table 2). Results of subgroup analysis based on the administration of conventional medications for insomnia, the severity of insomnia as per ISI score (scores of 15-21 or 22-28), and gender showed a significant difference between electroacupuncture and usual care; no difference was observed between electroacupuncture and sham-electroacupuncture at Week 4 regardless of the subgroup (Table S2). In addition, a similar result was observed in the per-protocol analysis including only patients who completed the treatment originally assigned to them (Table S3).

\section{Secondary Outcomes}

The mean PSQI scores in the three groups significantly decreased during the study period compared with those at baseline. Participants in the electroacupuncture group had significantly lower PSQI scores during the study period than those in the usual care group (Week 2: $\mathrm{P}=0.0048$, others: $\mathrm{P}<$ $0.0001)$. However, there were no differences in PSQI scores between electroacupuncture and sham-electroacupuncture groups ( $\mathrm{P}>0.05$, all). Sleep diary-derived SOL, WASO, TST, and SE significantly improved in the electroacupuncture and sham-acupuncture groups during the study period compared with the baseline values. Particularly, sleep diary-derived SE was significantly higher in the electroacupuncture group than in the sham-electroacupuncture $(\mathrm{P}=0.0397)$ and usual care groups $(\mathrm{P}=0.0002)$ at Week 4. Sleep diary-derived SOL

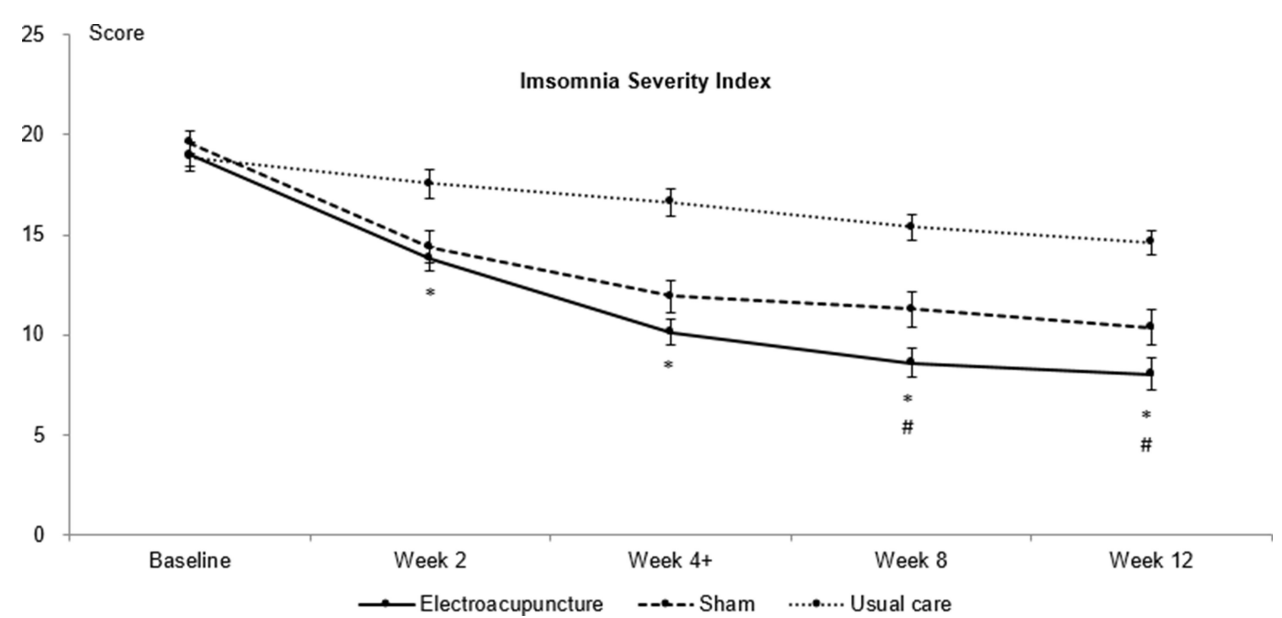

Figure 2 Mean Insomnia Severity Index scores of three groups during the study period *Significant difference between electroacupuncture and usual care, P<0.000I; ${ }^{\#}$ Significant difference between electroacupuncture and sham, $\mathrm{P}<0.05{ }^{+}{ }^{+}$The end of treatment. 


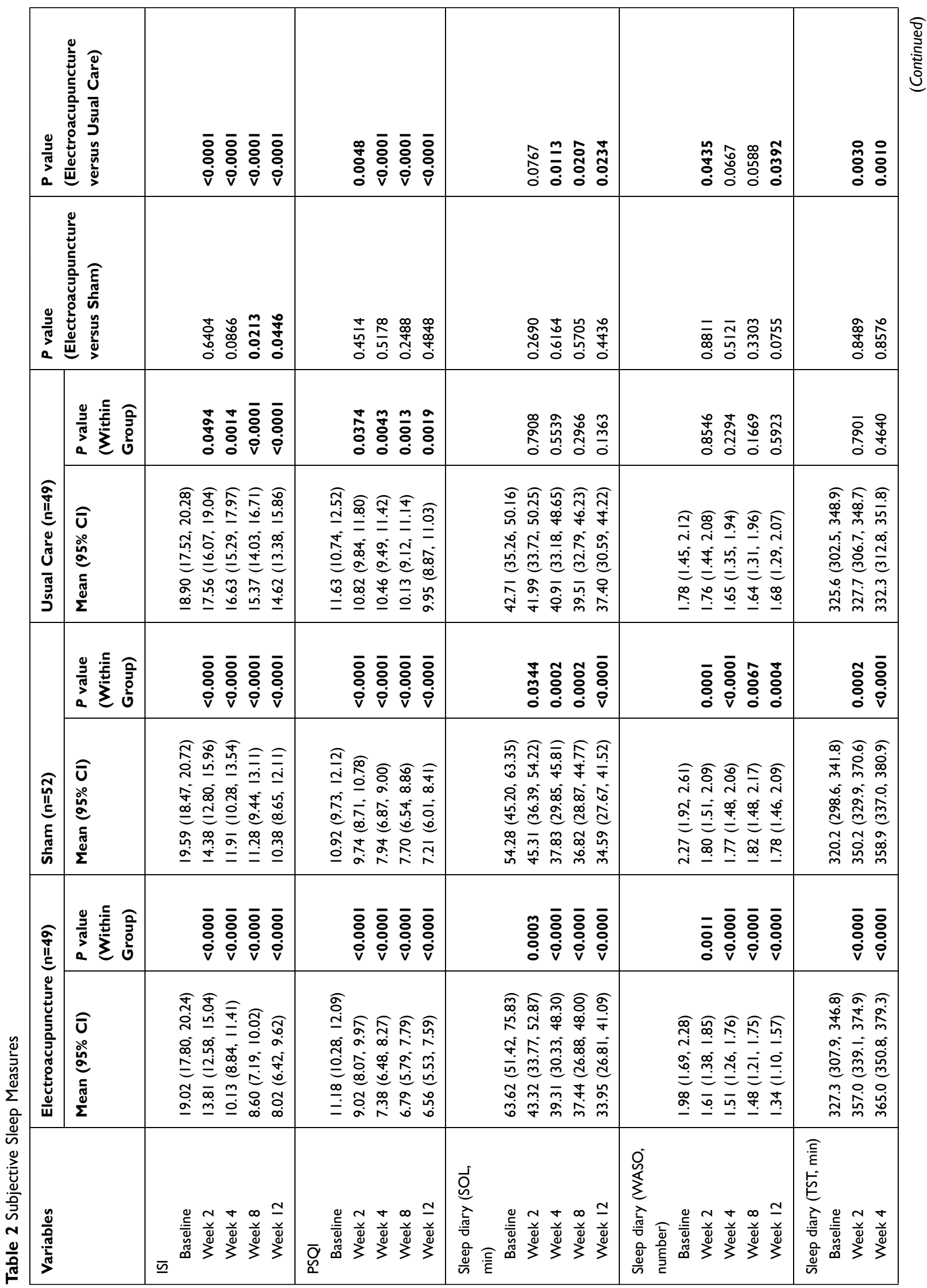




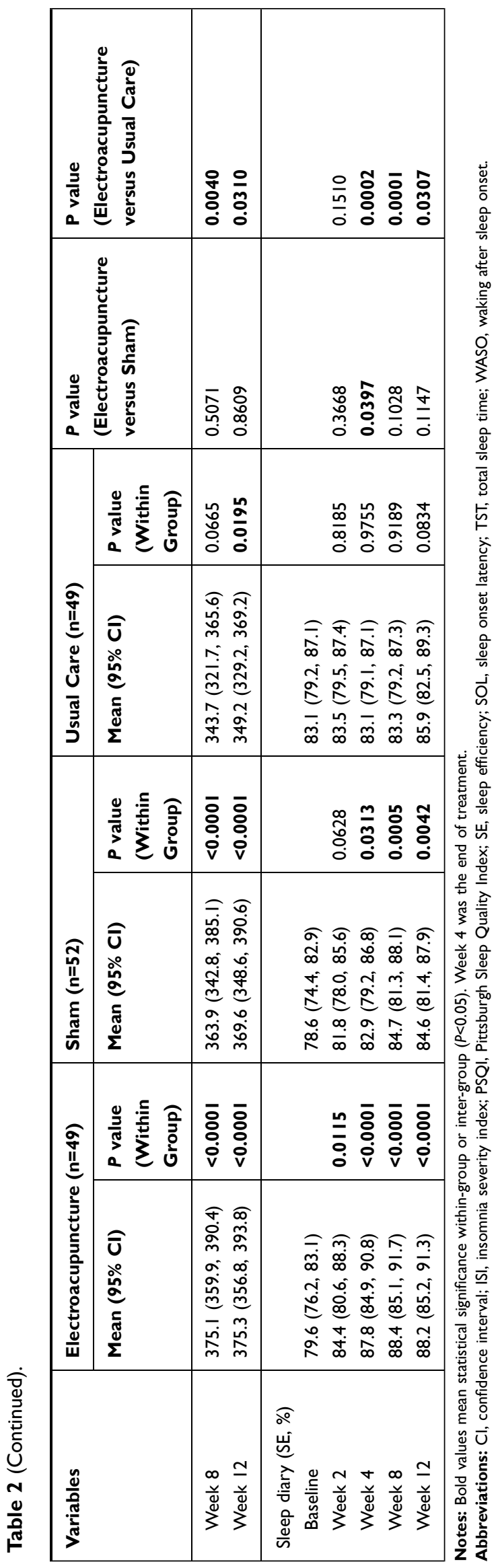

and TST were significantly improved in the electroacupuncture group compared with those in the usual care group at Week 4 ( $P=0.0113,0.0010)$; however, no differences were observed in these variables between the electroacupuncture and shamelectroacupuncture groups ( $\mathrm{P}>0.05$, all) (Table 2$)$.

The mean anxiety, depression, and total HADS scores significantly decreased after electroacupuncture and shamelectroacupuncture during the study period compared with those at baseline; however, these values showed no difference in the usual care group. The mean anxiety, depression, and total HADS scores significantly decreased in the electroacupuncture group compared with those in the usual care group during the study period; however, no significant differences were found in these parameters between electroacupuncture and sham-electroacupuncture groups. Further, the quality of life as measured by EQ-5D and EQVAS was significantly different between electroacupuncture and usual care groups only at our primary endpoint, which was Week 4. There were no differences in EQ-5D and EQ-VAS between electroacupuncture and shamelectroacupuncture groups. At Week 4, 24 patients (50\%) in the electroacupuncture group reported treatment success as per the PGIC scale, whereas 15 patients (31.91\%) in the sham-electroacupuncture group and 3 patients $(6.52 \%)$ in the usual care group reported treatment success (relative risk (RR) between electroacupuncture and shamelectroacupuncture: $1.57,95 \% \mathrm{CI}: 0.95$ to 2.59 ; $\mathrm{RR}$ between electroacupuncture and usual care: $7.67,95 \%$ CI: 2.48 to 23.73$)$. At Week 12,30 patients $(62.50 \%)$ in the electroacupuncture group reported treatment success, whereas 21 patients (44.68\%) in the shamelectroacupuncture group and 5 patients $(11.11 \%)$ in the usual care group reported treatment success (RR between electroacupuncture and sham: $1.40,95 \%$ CI: 0.95 to 2.06 ; RR between electroacupuncture and usual care: $5.63,95 \%$ CI: 2.39 to 13.23). No significant changes were observed in salivary melatonin and cortisol levels within each group during the study period and no difference was observed between electroacupuncture and sham-electroacupuncture and between electroacupuncture and usual care groups (Table 3).

\section{Safety Assessment}

In all, 106 AEs were reported during 1473 visits, and there was a statistically significant difference in the incidence of AEs among the three groups (electroacupuncture: 42 cases/626 visits [6.71\%], sham-electroacupuncture: 27 cases/616 visits [4.38\%], and usual care: 37 cases/231 


\begin{tabular}{|c|c|c|c|c|c|}
\hline 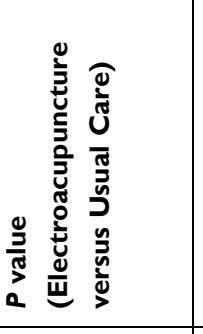 & 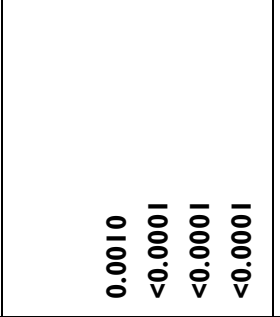 & 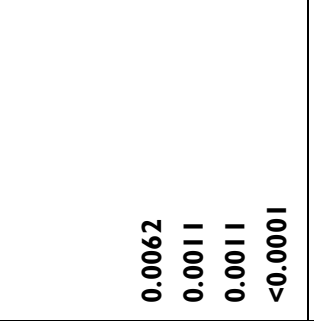 & 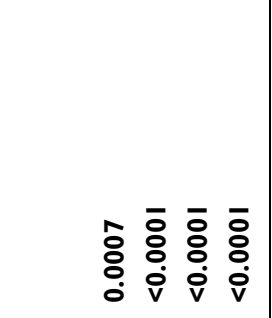 & 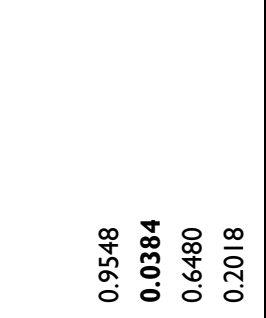 & 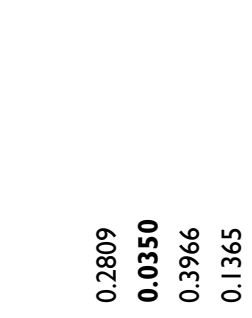 \\
\hline 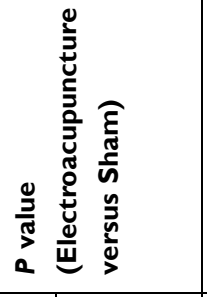 & 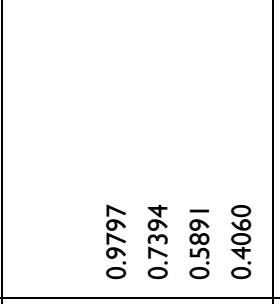 & 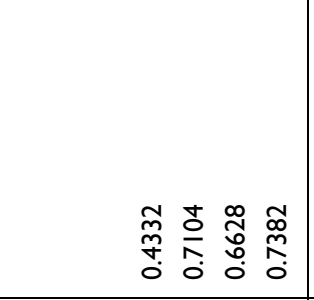 & 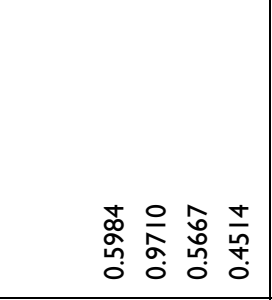 & 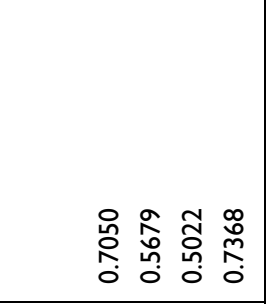 & 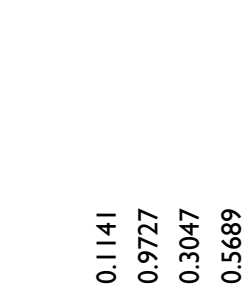 \\
\hline 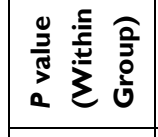 & 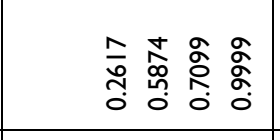 & 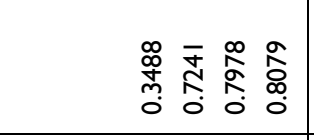 & 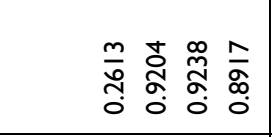 & 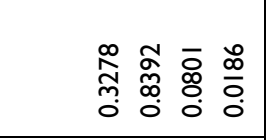 & 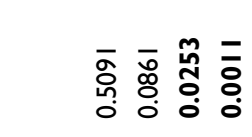 \\
\hline 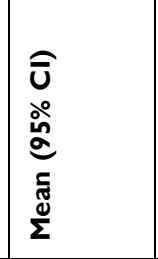 & 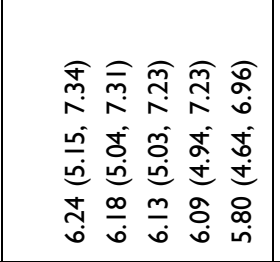 & 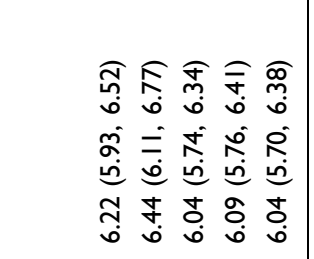 & 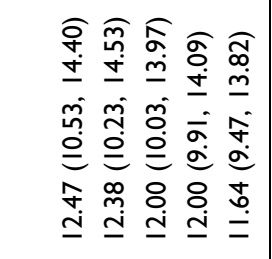 & 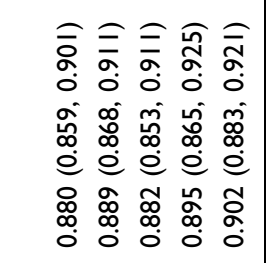 & 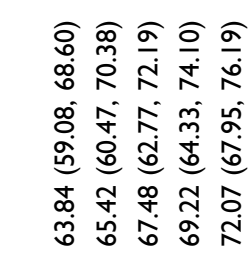 \\
\hline 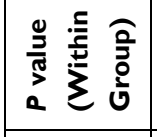 & 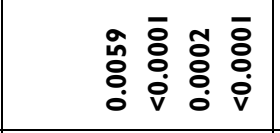 & 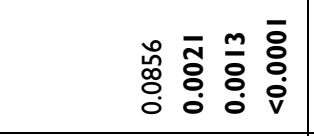 & 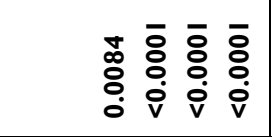 & 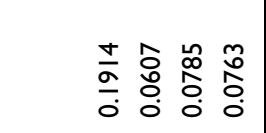 & 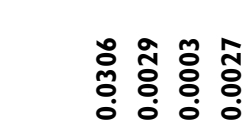 \\
\hline 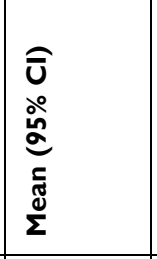 & 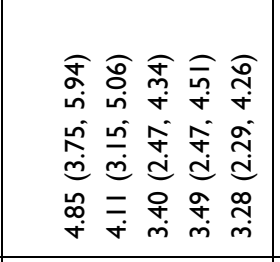 & 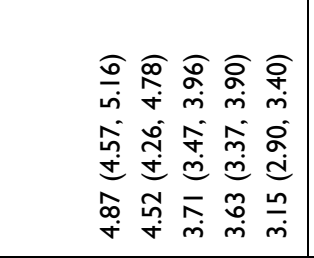 & 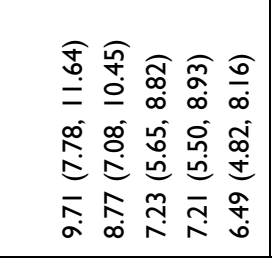 & 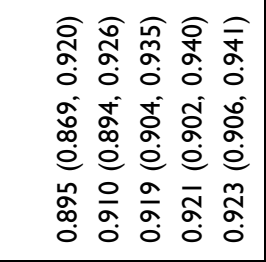 & 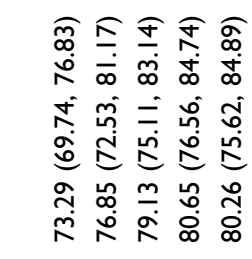 \\
\hline 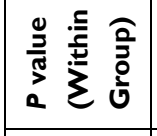 & 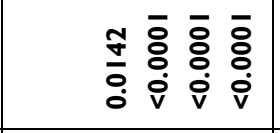 & 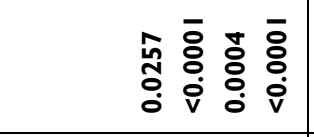 & 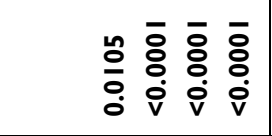 & 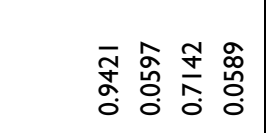 & 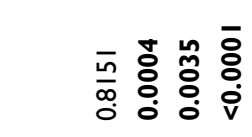 \\
\hline 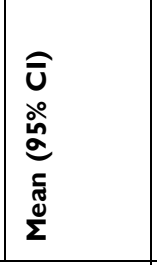 & 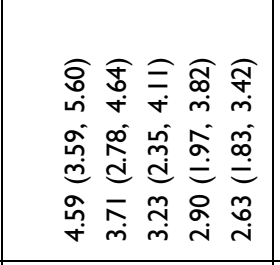 & 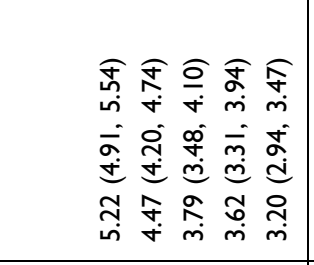 & 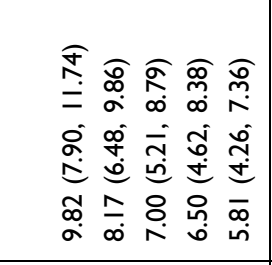 & 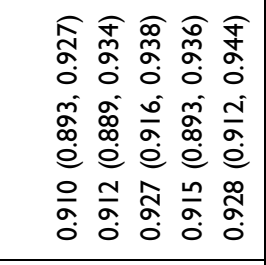 & 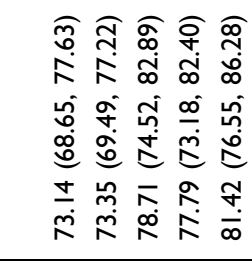 \\
\hline & 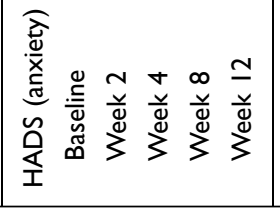 & 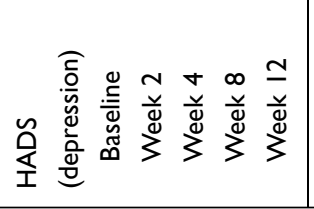 & 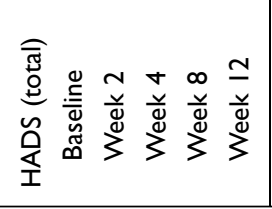 & 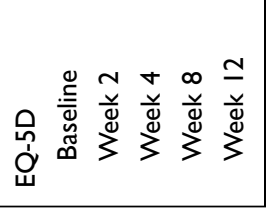 & 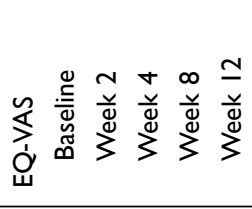 \\
\hline
\end{tabular}




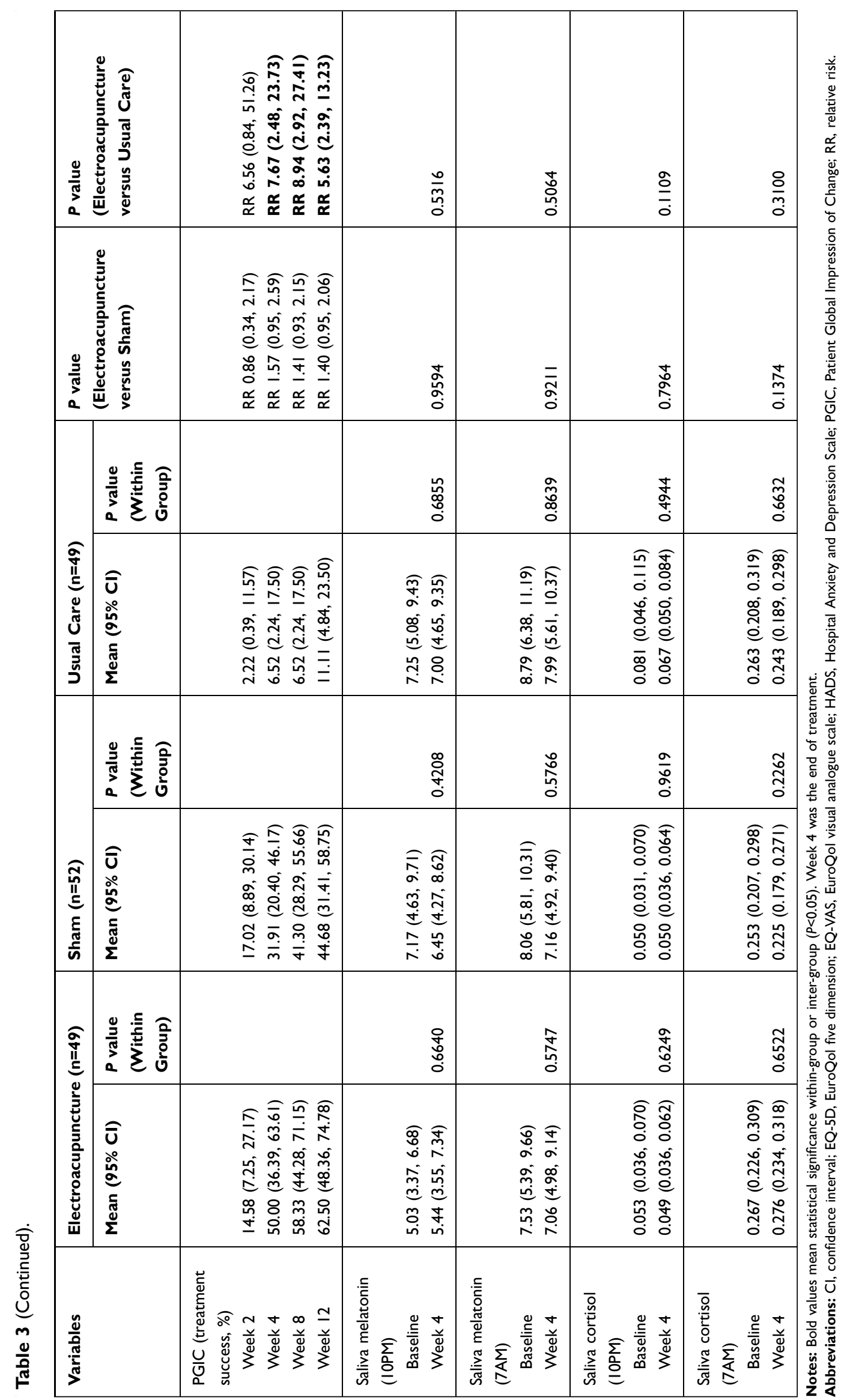


visits $[16.02 \%] ; \mathrm{P}<0.0001)$. The most common $\mathrm{AE}$ was upper respiratory tract infection $(58.49 \%)$, followed by dyspepsia (14.15\%) and headache (4.72\%) (Table 4). There was no AE related to treatment, and no serious AE was reported. All AEs improved spontaneously during the study period and there were no study drop-outs because of AEs. In addition, no significant differences were observed in laboratory test results before and after treatment in all groups (Table S4).

\section{Blinding Test}

At Week 1 post-treatment, 36 of 49 participants in the electroacupuncture group (new BI: $0.612,95 \%$ CI: 0.418 to 0.807 ) versus 31 of 48 participants in sham-electroacupuncture group (new BI: $-0.479,95 \%$ CI: -0.695 to -0.263 ) guessed their treatment to be real electroacupuncture. At Week 4, these numbers were 36 of 48 participants in the electroacupuncture group (new BI: $0.646,95 \%$ CI: 0.459 to 0.833 ) and 27 of 47 participants in the sham-electroacupuncture group (new BI: $-0.255,95 \%$ CI: -0.516 to 0.005 ) (Table S5). This result implies that blinding was appropriately maintained in the sham-electroacupuncture group during the study period.

\section{Discussion}

The findings of this study indicate that electroacupuncture leads to a greater improvement in insomnia severity as measured by ISI and PSQI in moderate-to-severe insomnia patients (ISI score of at least 15) compared with usual care after 4 weeks of treatment. In addition, the significant differences were well-maintained until Week 12 (8-week follow-up after the final intervention). Considering that the minimal clinically important differences (MCIDs) in ISI and PSQI scores are 6 and 3 points for insomnia patients, respectively, ${ }^{43,44}$ a mean decrease of 8.83 and 3.73 in ISI and PSQI scores, respectively, at Week 4 demonstrated that electroacupuncture had a clinically meaningful benefit in improving the sleep quality in these patients. The mean difference in ISI score after 4 weeks of electroacupuncture in this study was similar to that reported in a previous study measuring ISI score difference after 6 weeks of zolpidem plus CBT-I, although our study included patients with more severe insomnia; thus, a direct comparison cannot be made. ${ }^{45}$ The PSQI score difference and baseline scores were also similar to those reported in a previous study measuring PSQI score difference after 6 weeks of acupuncture or estazolam compared with baseline. ${ }^{46}$ In addition, electroacupuncture significantly improved sleep diary-derived variables, anxious and depressive moods as measured by HADS, and health-related quality of life as measured by EQ-5D and EQ-VAS compared with usual care.

There was no significant difference in ISI score between electroacupuncture and sham-electroacupuncture groups at Week 4 , which was our primary endpoint, although there was a significant difference in sleep diary-derived SE between the two groups. Therefore, the results of this study cannot conclusively prove that electroacupuncture had a better effect than sham-electroacupuncture. Some previous studies have shown significant differences in ISI scores between acupuncture and sham-acupuncture. ${ }^{20,22}$ However, these differ from our study because one of these studies included insomnia patients with major depressive disorders, ${ }^{20}$ and another used the same acupoints without electrical stimulation in both acupuncture and shamacupuncture groups. ${ }^{22}$ Thus, the results of the current study can be interpreted based on the following ways. First, various factors are involved in acupuncture treatment during the procedure, eg, the tool (needle), psychology (both of the patient and the practitioner), technique, and education level of the practitioner. ${ }^{47}$ Some studies have suggested that a placebo response driven by factors such as interpersonal interactions plays an important role in

Table 4 Number of Adverse Events

\begin{tabular}{|l|l|l|l|l|}
\hline Adverse Event & Electroacupuncture (626 Visits) & Sham (616 Visits) & Usual Care (23I Visits) & Total \\
\hline Overall & 42 & 27 & 37 & 106 \\
Serious adverse event & 0 & 0 & 0 & 0 \\
Upper respiratory infection & $24(57.14 \%)$ & $13(48.15 \%)$ & $25(67.57 \%)$ & $62(58.49 \%)$ \\
Dyspepsia & $7(16.67 \%)$ & $4(14.81 \%)$ & $4(10.81 \%)$ & $15(14.15 \%)$ \\
Headache & $I(2.38 \%)$ & $4(14.81 \%)$ & 0 & $5(4.72 \%)$ \\
Low back pain & $I(2.38 \%)$ & 0 & $1(2.70 \%)$ & $2(1.89 \%)$ \\
Pruritus & $I(2.38 \%)$ & $1(3.70 \%)$ & 0 & $2(1.89 \%)$ \\
Others & $8(19.05 \%)$ & $5(18.52 \%)$ & $7(18.92 \%)$ & $20(18.87 \%)$ \\
\hline
\end{tabular}


certain disorders, eg, traumatic brain injury, migraine, chronic pain, depression, and insomnia. ${ }^{48,49}$ Therefore, there is a possibility that other related factors except the sham needle may have influenced the results. Second, although there was no difference between electroacupuncture and sham-electroacupuncture in terms of ISI scores at Week 4, ISI scores were significantly different between the two groups at Weeks 8 and 12. Therefore, the trial period may not have been long enough to see the effect of electroacupuncture compared with sham-electroacupuncture. Accordingly, it is necessary to conduct a long-term trial to assess the difference between these two modalities in the future.

Various experimental and clinical studies have been conducted to elucidate the mechanism of acupuncture treatment for treating insomnia. These studies suggest that neurotransmitters including serotonin, glutamate, gamma-aminobutyric acid, and melatonin are involved in the mechanism of acupuncture treatment for insomnia. ${ }^{50-53}$ In particular, previous studies have shown the potential of acupuncture and electroacupuncture for treating insomnia by regulating melatonin and cortisol levels, ${ }^{54-56}$ although our study showed no significant changes in the levels of these factors after electroacupuncture. However, because of the differences in the acupuncture treatment method and treatment period, further studies are needed to reach a definitive conclusion. Other experimental studies have suggested that acupuncture improved insomnia by regulating the hypothalamic-pituitary-adrenal axis. ${ }^{55,56}$ Furthermore, although the underlying pathophysiological mechanisms are poorly understood, genome-wide association analyses have identified 57 loci associated with insomnia. ${ }^{57}$ Several animal experiments have reported that acupuncture treatment for insomnia may be associated with mRNA expression or gene transcription, and clinical studies targeting changes in intestinal microbiome after acupuncture for insomnia have been conducted. ${ }^{58,59}$ Therefore, the delayed response of acupuncture treatment observed in our study may have resulted from genetic variations occurring as a cumulative effect of acupuncture treatment. However, to the best of our knowledge, the mechanisms of acupuncture treatment for treating insomnia still remain unclear and further experimental studies are warranted to reveal the underlying mechanisms.

The strength of this trial is the successful blinding of the sham-electroacupuncture group. Previous studies mostly lacked blinding assessment; ${ }^{20-22}$ therefore, their results may be biased depending on whether or not blinding was maintained. We evaluated whether the blinding was well maintained during the study period through two blinding tests conducted after the first and the last treatment. The results in the two tests showed that blinding in the shamelectroacupuncture group was well-maintained throughout the study period. This result is particularly encouraging, given that the individuals in East Asia, including Korea, are very familiar with acupuncture. In addition, the drop-out rate during the study period was as low as $13.4 \%$ in the shamelectroacupuncture group. For the sham-electroacupuncture group, we inserted a validated sham-acupuncture device, PSD, at non-acupoints in both the arms and legs and used a de-activated electroacupuncture device that made the same beeping sound and light indications as in the electroacupuncture group. Additionally, we treated all participants in a treatment room environment similar to a general Korean medicine hospital or clinic, and the acupuncture therapist limited conversations not related to the treatment with the participants. These factors may have contributed to maintaining the blindness among participants during the study period in the sham-electroacupuncture group, resulting in a low drop-out rate. Another strength of our study is the superiority of the study design that evaluates both the efficacy and effectiveness of electroacupuncture by comparing it with sham-electroacupuncture and usual care, respectively. Previous acupuncture studies on insomnia have often used non-penetrating sham acupuncture at real acupoints. However, non-penetrating sham acupuncture at real acupoints also can lead to a "de qi" sensation that is important in creating a positive clinical outcome. ${ }^{31,60,61}$ Additionally, sham acupoints beside the real acupoints can also be sensitized by an increased expression of nociceptive substances. ${ }^{62}$ We used validated non-penetrating sham acupuncture at heterosegmental non-acupoints to minimize any physiological effect. Furthermore, efficacy trials without a usual care arm have been termed as a fundamental flaw because it is only meaningful to compare two interventions if they have been shown to be superior to usual care. ${ }^{63}$

However, there were some limitations to our study. First, the baseline characteristics of the participants showed that there were significantly fewer women in the usual care group. Although there is less concern on baseline characteristic imbalance if a trial has a large sample size and uses a truly random process protected with concealed allocation in RCTs, ${ }^{64}$ it has been reported that females are associated with a $40 \%$ increased risk of developing insomnia compared with males. ${ }^{65,66}$ To investigate the effect of baseline gender differences on effect size, we analyzed the effect size using baseline gender as a covariate. As a result, it was confirmed that gender did not significantly affect the results 
(Table S6). In addition, we conducted a subgroup analysis based on gender for ISI score and observed no significant difference from the overall analysis results. Nonetheless, it is necessary to design a stratified clinical trial considering gender differences to prevent the possibility of such a bias in the future. Second, we could not measure the sleep patterns of participants using objective measures such as actigraphy or polysomnography. Although polysomnography is considered the gold standard in sleep research, it is not always possible to conduct in actual clinical studies because of limitations such as costs involved, institution's infrastructure, and patients' inconvenience to be hospitalized for the measurement. Furthermore, correlations between ISI score, which was our primary outcome, and polysomnographic measures have been found to be significant in several studies. ${ }^{32,67}$ Future studies should be conducted using objective measures including polysomnographic measurement.

\section{Conclusion}

Results of the current trial provide supportive evidence that electroacupuncture can be an effective, safe, and welltolerated non-pharmacological intervention for insomnia, using validated placebo control and validated subjective questionnaires. Further long-term studies with objective sleep measures are needed for a more definitive conclusion.

\section{Data Sharing Statement}

The data that support the findings of this study are available from the corresponding author (Joo-Hee Kim) upon reasonable request. Additionally, the individualdeidentified participant data are available after contacting the corresponding author via email. The data will be available immediately following publication without an end date.

\section{Acknowledgments}

This work was supported by the Korea Institute of Oriental Medicine (K16122 and KSN1522120).

\section{Disclosure}

The authors declare that they have no conflicts of interest.

\section{References}

1. American Psychiatric Association. Sleep-wake disorders. 5th. In: Diagnostic and Statistical Manual of Mental Disorders. Arlington, VA: American Psychiatric Association; 2013.
2. Nowicki Z, Grabowski K, Cubala WJ, et al. Prevalence of self-reported insomnia in general population of Poland. Psychiatr Pol. 2016;50(1):165-173. doi:10.12740/PP/58771

3. Ohayon MM. Epidemiology of insomnia: what we know and what we still need to learn. Sleep Med Rev. 2002;6(2):97-111. doi:10.1053/ smrv.2002.0186

4. Bertisch SM, Pollock BD, Mittleman MA, et al. Insomnia with objective short sleep duration and risk of incident cardiovascular disease and all-cause mortality: Sleep Heart Health Study. Sleep. 2018;41(6). doi:10.1093/sleep/zsy047.

5. Katz DA, McHorney CA. The relationship between insomnia and health-related quality of life in patients with chronic illness. $J$ Fam Pract. 2002;51(3):229-235.

6. Vgontzas AN, Liao D, Pejovic S, Calhoun S, Karataraki M, Bixler EO. Insomnia with objective short sleep duration is associated with type 2 diabetes: a population-based study. Diabetes Care. 2009;32(11):1980-1985. doi:10.2337/dc09-0284

7. Woznica AA, Carney CE, Kuo JR, Moss TG. The insomnia and suicide link: toward an enhanced understanding of this relationship. Sleep Med Rev. 2015;22:37-46. doi:10.1016/j.smrv.2014.10.004

8. Sateia MJ, Buysse DJ, Krystal AD, Neubauer DN, Heald JL. Clinical practice guideline for the pharmacologic treatment of chronic insomnia in adults: an American Academy of Sleep Medicine Clinical Practice Guideline. J Clin Sleep Med. 2017;13(2):307-349.

9. Schutte-Rodin S, Broch L, Buysse D, Dorsey C, Sateia M. Clinical guideline for the evaluation and management of chronic insomnia in adults. J Clin Sleep Med. 2008;4(5):487-504.

10. Baron KG, Hooker S. Next steps for patients who fail to respond to cognitive behavioral therapy for insomnia (CBT-I): the perspective from behavioral sleep medicine psychologists. Curr Sleep Med Rep. 2017;3(4):327-332. doi:10.1007/s40675-017-0096-x

11. Zachariae R, Lyby MS, Ritterband LM, O’Toole MS. Efficacy of internet-delivered cognitive-behavioral therapy for insomnia A systematic review and meta-analysis of randomized controlled trials. Sleep Med Rev. 2016;30:1-10. doi:10.1016/j.smrv.2015.10.004

12. Buscemi N, Vandermeer B, Friesen C, et al. The efficacy and safety of drug treatments for chronic insomnia in adults: a meta-analysis of RCTs. J Gen Intern Med. 2007;22(9):1335-1350. doi:10.1007/ s11606-007-0251-z

13. Vermeeren A. Residual effects of hypnotics: epidemiology and clinical implications. CNS Drugs. 2004;18(5):297-328. doi:10.2165/ 00023210-200418050-00003

14. National Institute of Health. National institutes of health state of the science conference statement on manifestations and management of chronic insomnia in adults, June 13-15, 2005. Sleep. 2005;28 (9):1049-1057.

15. Bertisch SM, Wells RE, Smith MT, McCarthy EP. Use of relaxation techniques and complementary and alternative medicine by American adults with insomnia symptoms: results from a national survey. J Clin Sleep Med. 2012;8(6):681-691.

16. Eisenberg DM, Davis RB, Ettner SL, et al. Trends in alternative medicine use in the United States, 1990-1997: results of a followup national survey. JAMA. 1998;280(18):1569-1575. doi:10.1001/ jama.280.18.1569

17. Zhang Y, Lao L, Chen H, Ceballos R. Acupuncture use among american adults: what acupuncture practitioners can learn from national health interview survey 2007? Evid Based Complement Alternat Med. 2012;2012:710750. doi:10.1155/2012/710750

18. Lee HG, Jung DJ, Choi YM, et al. A systematic literature review on clinical research in human utilized electroacupuncture in Korea. $J$ Acupunct Res. 2013;30(5):139-153. doi:10.13045/acupunct. 2013053

19. Xu H, Shi Y, Xiao Y, et al. Efficacy comparison of different acupuncture treatments for primary insomnia: a bayesian analysis. Evid Based Complement Alternat Med. 2019;2019:8961748. doi:10.1155/ 2019/8961748 
20. Yeung WF, Chung KF, Tso KC, Zhang SP, Zhang ZJ, Ho LM. Electroacupuncture for residual insomnia associated with major depressive disorder: a randomized controlled trial. Sleep. 2011;34 (6):807-815. doi:10.5665/SLEEP.1056

21. Yeung WF, Chung KF, Zhang SP, Yap TG, Law AC. Electroacupuncture for primary insomnia: a randomized controlled trial. Sleep. 2009;32(8):1039-1047.

22. Yin X, Gou M, Xu J, et al. Efficacy and safety of acupuncture treatment on primary insomnia: a randomized controlled trial. Sleep Med. 2017;37:193-200. doi:10.1016/j.sleep.2017.02.012

23. Qaseem A, Kansagara D, Forciea MA, Cooke M, Denberg TD. Management of chronic insomnia disorder in adults: a Clinical Practice Guideline From the American College of Physicians. Ann Intern Med. 2016;165(2):125-133. doi:10.7326/M15-2175

24. Kim SP, Kim JH, Kim BK, et al. Electroacupuncture for insomnia disorder: study protocol for a randomized controlled trial. Trials. 2017;18(1):177. doi:10.1186/s13063-017-1922-7

25. MacPherson H, Altman DG, Hammerschlag R, et al. Revised STandards for Reporting Interventions in Clinical Trials of Acupuncture (STRICTA): extending the CONSORT statement. $J$ Altern Complement Med. 2010;16(10):St1-14. doi:10.1089/acm.2010.1610

26. Moher D, Hopewell S, Schulz KF, et al. CONSORT 2010 explanation and elaboration: updated guidelines for reporting parallel group randomised trials. $B M J$. 2010;340:c869. doi:10.1136/bmj.c869

27. Zhao K. Acupuncture for the treatment of insomnia. Int Rev Neurobiol. 2013;111:217-234.

28. Yeung WF, Chung KF, Leung YK, Zhang SP, Law ACK. Traditional needle acupuncture treatment for insomnia: a systematic review of randomized controlled trials. Sleep Med. 2009;10(7):694-704. doi:10.1016/j.sleep.2008.08.012

29. Korean Acupuncture and Moxibustion Medicine Society Textbook Compilation Committee. The Acupuncture and Moxibustion Medicine. Paju: Jipmoondang; 2012.

30. Gong X-I, Pan Z-H, Shen Y, Wang S. Blinding effect of non-penetrating sham needle in the randomized controlled trials of acupuncture: a systematic review. J Acupunct Tuina Sci. 2014;12 (1):8-11. doi:10.1007/s11726-014-0738-1

31. Park J, White A, Stevinson C, Ernst E, James M. Validating a new non-penetrating sham acupuncture device: two randomised controlled trials. Acupunct Med. 2002;20(4):168-174.

32. Bastien $\mathrm{CH}$, Vallieres A, Morin CM. Validation of the insomnia severity index as an outcome measure for insomnia research. Sleep Med. 2001;2(4):297-307. doi:10.1016/S1389-9457(00)00065-4

33. Cho YW, Song ML, Morin CM. Validation of a Korean version of the insomnia severity index. J Clin Neurol. 2014;10(3):210-215. doi:10.3988/jcn.2014.10.3.210

34. Buysse DJ, Reynolds CF 3rd, Monk TH, Berman SR, Kupfer DJ. The Pittsburgh Sleep Quality Index: a new instrument for psychiatric practice and research. Psychiatry Res. 1989;28(2):193-213.

35. Sohn SI, Kim DH, Lee MY, Cho YW. The reliability and validity of the Korean version of the Pittsburgh sleep quality index. Sleep Breath. 2012;16(3):803-812. doi:10.1007/s11325-011-0579-9

36. Oh SM, Min KJ, Park DB. A study on the standardization of the hospital anxiety and depression scale for Koreans: a comparison of normal, depressed and anxious groups. J Korean Neuropsychiatr Assoc. 1999;38(2):289.

37. Kim MH, Cho YS, Uhm WS, Kim S, Bae SC. Cross-cultural adaptation and validation of the Korean version of the EQ-5D in patients with rheumatic diseases. Qual Life Res. 2005;14(5):1401-1406. doi:10.1007/s11136-004-5681-z

38. Rabin R, de Charro F. EQ-5D: a measure of health status from the EuroQol Group. Ann Med. 2001;33(5):337-343. doi:10.3109/ 07853890109002087

39. Hurst H, Bolton J. Assessing the clinical significance of change scores recorded on subjective outcome measures. $J$ Manipulative Physiol Ther. 2004;27(1):26-35. doi:10.1016/j.jmpt.2003.11.003
40. Huang W, Kutner N, Bliwise DL. Autonomic activation in insomnia: the case for acupuncture. J Clin Sleep Med. 2011;7(1):95-102.

41. Park JH, Lee JH, Lee S, Shin JY, Kim TH. Adverse events related to electroacupuncture: a systematic review of single case studies and case series. Acupunct Med. 2020;964528420920287.

42. Bang H, Ni L, Davis CE. Assessment of blinding in clinical trials. Control Clin Trials. 2004;25(2):143-156. doi:10.1016/j.cct.2003.10.016

43. Hughes CM, McCullough CA, Bradbury I, et al. Acupuncture and reflexology for insomnia: a feasibility study. Acupunct Med. 2009;27 (4):163-168. doi:10.1136/aim.2009.000760

44. Yang M, Morin CM, Schaefer K, Wallenstein GV. Interpreting score differences in the Insomnia Severity Index: using health-related outcomes to define the minimally important difference. Curr Med Res Opin. 2009;25(10):2487-2494. doi:10.1185/03007990903167415

45. Morin CM, Vallières A, Guay B, et al. Cognitive behavioral therapy, singly and combined with medication, for persistent insomnia: a randomized controlled trial. JAMA. 2009;301(19):2005-2015. doi:10.1001/jama.2009.682

46. Guo J, Wang LP, Liu CZ, et al. Efficacy of acupuncture for primary insomnia: a randomized controlled clinical trial. Evid Based Complement Alternat Med. 2013;2013:163850. doi:10.1155/2013/ 163850

47. Wang Y-R, Zhao J-P, Hao D-F. Is sham acupuncture a real placebo: skeptical for sham acupuncture. World $J$ Acupunct Moxibustion. 2017;27(2):1-5. doi:10.1016/S1003-5257(17)30110-1

48. Bernstein MH, Brown WA. The placebo effect in psychiatric practice. Curr Psychiatr. 2017;16(11):29-34.

49. Polich G, Iaccarino MA, Kaptchuk TJ, Morales-Quezada L, Zafonte R. Placebo effects in traumatic brain injury. J Neurotrauma. 2018;35(11):1205-1212. doi:10.1089/neu.2017.5506

50. Liu Z, Chen X, Gao Y, et al. Involvement of GluR2 up-regulation in neuroprotection by electroacupuncture pretreatment via cannabinoid CB1 receptor in mice. Sci Rep. 2015;5:9490. doi:10.1038/srep09490

51. Spence DW, Kayumov L, Chen A, et al. Acupuncture increases nocturnal melatonin secretion and reduces insomnia and anxiety: a preliminary report. J Neuropsychiatry Clin Neurosci. 2004;16 (1):19-28. doi:10.1176/jnp.16.1.19

52. Yang JL, Zhang R, Du L, Yang YS, Liu XC. Clinical observation on the neurotransmitters regulation in patients of insomnia differentiated as yang deficiency pattern treated with warm acupuncture and auricular point sticking therapy. Zhongguo Zhen Jiu. 2014;34 (12):1165-1168.

53. Zhou YL, Gao XY, Wang PY, Ren S. Effect of acupuncture at different acupoints on expression of hypothalamic GABA and GABA(A) receptor proteins in insomnia rats. Acupunct Res. 2012;37(4):302-307.

54. Nordio M, Romanelli F. Efficacy of wrists overnight compression (HT 7 point) on insomniacs: possible role of melatonin? Minerva Med. 2008;99(6):539-547.

55. Park HJ, Park HJ, Chae Y, Kim JW, Lee H, Chung JH. Effect of acupuncture on hypothalamic-pituitary-adrenal system in maternal separation rats. Cell Mol Neurobiol. 2011;31(8):1123-1127. doi:10.1007/s10571-011-9718-x

56. You W, Shi YJ, Han YJ, Jia BH, Tu Y. Effect of electroacupuncture of "Baihui" (GV 20)-"Yintang" (EX-HN 3) on the expression of glucocorticoid and glucocorticoid receptor mRNA of the chronic stress model rats. Acupunct Res. 2010;35(4):261-266.

57. Huangfu YR, Peng W, Guo BJ, et al. Effects of acupuncture in treating insomnia due to spleen-stomach disharmony syndrome and its influence on intestinal microbiome: study protocol for a randomized controlled trial. J Integr Med. 2019;17(3):161-166. doi:10.1016/j.joim.2019.01.007

58. Wei XR, Wei GW, Zheng XN, et al. Effect of acupuncture stimulation of different acupoint combinations on sleep and expression of circadian clock and Bmal 1 genes in hypothalamus of insomnia rats. Acupunct Res. 2017;42(5):429-433. 
59. Zheng XN, Wu XF, Guo X, et al. Manual acupuncture stimulation of paired acupoints can relieve sleep disorder possibly by upregulating pineal melatonin protein and its receptor mRNA levels in the suprachiasmatic nucleus in insomnia rats. Acupunct Res. 2018;43 (6):360-364.

60. Lee H, Bang H, Kim Y, et al. Non-penetrating sham needle, is it an adequate sham control in acupuncture research? Complement Ther Med. 2011;19(Suppl 1):S41-48. doi:10.1016/j.ctim.2010.12.002

61. Mao JJ, Farrar JT, Armstrong K, Donahue A, Ngo J, Bowman MA. De qi: chinese acupuncture patients' experiences and beliefs regarding acupuncture needling sensation-an exploratory survey. Acupunct Med. 2007;25(4):158-165. doi:10.1136/aim.25.4.158

62. He W, Wang XY, Shi H, et al. Cutaneous neurogenic inflammation in the sensitized acupoints induced by gastric mucosal injury in rats. BMC Complement Altern Med. 2017;17(1):141.
63. Thompson BT, Schoenfeld D. Usual care as the control group in clinical trials of nonpharmacologic interventions. Proc Am Thorac Soc. 2007;4(7):577-582. doi:10.1513/pats.200706-072JK

64. Elkins MR. Assessing baseline comparability in randomised trials. J Physiother. 2015;61(4):228-230. doi:10.1016/j.jphys.2015.07.005

65. Drake CL, Pillai V, Roth T. Stress and sleep reactivity: a prospective investigation of the stress-diathesis model of insomnia. Sleep. 2014;37(8):1295-1304. doi:10.5665/sleep.3916

66. Zhang B, Wing YK. Sex differences in insomnia: a meta-analysis. Sleep. 2006;29(1):85-93. doi:10.1093/sleep/29.1.85

67. Sadeghniiat-Haghighi K, Yazdi Z, Firoozeh M. Comparison of two assessment tools that measure insomnia: the insomnia severity index and polysomnography. Indian J Psychol Med. 2014;36(1):54-57. doi:10.4103/0253-7176.127251

\section{Publish your work in this journal}

Nature and Science of Sleep is an international, peer-reviewed, open access journal covering all aspects of sleep science and sleep medicine, including the neurophysiology and functions of sleep, the genetics of sleep, sleep and society, biological rhythms, dreaming, sleep disorders and therapy, and strategies to optimize healthy sleep.
The manuscript management system is completely online and includes a very quick and fair peer-review system, which is all easy to use. Visit http://www.dovepress.com/testimonials.php to read real quotes from published authors. 